IP Periodica Polytechnica Mechanical Engineering

RESEARCH ARTICLE

\section{Mass Flux Distribution Measurements and Visualizations of a Fluid Sheet Generated by a Partially Immersed Dip-Lubricated Gear}

\author{
Zsolt Várhegyi ${ }^{1}$, Gergely Kristóf ${ }^{1 *}$
}

Received 16 October 2014; accepted after revision 20 January 2016

\begin{abstract}
A simplified model of gearbox lubrication flow is investigated. A metric gear with module size $4 \mathrm{~mm}$, tooth number 38, tip radius $80.25 \mathrm{~mm}$ and width $40 \mathrm{~mm}$, is partially immersed and rotated between 0-12 1/s frequencies in room temperature distilled water. Flash and laser sheet visualizations of the global flow field and the boundary layer are presented. Resolved mass flow distributions in the fluid sheet above the water surface are obtained with in-situ liquid trapping (bucketing). The relation between the circumferential velocity and integrated mass flux of the fluid sheet is found to be linear above a critical speed, for which an analytical formula is given. Using dimensional analysis, we show that the relation between the mass flux and gear speed signifies a Reynolds-number independent behavior of the boundary layer around the immersed part of the gear.
\end{abstract}

\section{Keywords}

dip lubrication, gearbox, two-phase, fluid sheet, Reynoldsindependent, boundary layer

\footnotetext{
${ }^{1}$ Department of Fluid Mechanics, Faculty of Mechanical Engineering, Budapest University of Techology and Economics,

H-1521 Budapest, P.O.B. 91, Hungary

*Corresponding author, e-mail: kristof@ara.bme.hu
}

\section{Introduction}

Investigation of the flow in a gearbox of dip-lubricated transmission is a challenging task both numerically and experimentally. Such flows include two or more phases: the lubricant liquid, air and solved and insoluble additives, bubble and drop formation and coalescence, thin films, turbulence, heat and mass transport; viscoelasticity and drastic changes in other transport properties of the lubricant due to heat flash and pressure rise in the vicinity of the contact region.

Despite the complex flow phenomena the designer of the transmission may very well be interested only in the power losses due to churning of the oil and the effectiveness of the dip-lubrication by verifying that the splashing oil reaches some specific locations, a gear or a bearing, which was originally not immersed in the oil bath in standstill.

Several authors report successful simulations. In [1], singlephase FVM VOF $k-\varepsilon$ simulations using dynamic mesh in a twogear transmission gearbox fully immersed in oil are presented. The authors of [2] show a two-phase flow simulation in a jetlubricated bevel gear-pair in auxiliary equipment of a jet engine using FVM, VOF and a dynamic mesh. Two-phase FVM VOF $k-\varepsilon$ simulations are presented in [3-7], and CFD simulation of a full 7-speed dual clutch automotive transmission including jet and dip lubrication is presented in [8]. Having such a task as an industrial problem in the early design phase, a great difficulty is encountered when one would like to simulate such complex two-phase flows reliably within realistic time and computational constraints. In [9], the authors use a moving reference frame to obtain a quasi-steady CFD simulation of a single phase flow around a single gear. in order to have a 50-fold reduction in computational demand. In [10], a method of dimensional analysis to determine the dominant heat generation mechanism between contact heating or churning losses and then a computational method for thermohydrodynamic simulation decoupling the time scales for heat conduction in the gear and the unsteady turbulent two phase flow around a single jet lubricated gear are presented.

In the above examples, mesh or turbulence or two phase model independence studies are usually not shown. This brings 
up the general question: which simplifications are justified to meet constraints while keeping solution uncertainties below an acceptable level when simulating gearbox lubrication flows. The real difficulty is encountered while one would like to validate the simulation in detail, using experimental measurements.

The experimental investigation of oil flow in transmission housings has a longer past than CFD since bed tests were the usual method before the advent of numerical simulations. For such a test, the gearbox would have to be manufactured and then modified to enable access for optical and/or other measurement methods. While this method is extremely labor- and cost-intensive, in case of a new design which exists only on the drawing table, it is impossible.

Many measurement results are available for simplified geometries for the churning power losses in direct data form [11-13], or in the form of correlations, based on dimensional and/or nondimensional parameters. A review of the older literature in this regard is found in [14]. More recent investigations on the power losses on a partially immersed single spur gear in a rectangular tank show that the previous correlations can bear several hundred percent of relative errors compared to experiments [15]. In [16] the authors investigated the effect axial and radial clearances on the dimensionless torque coefficient and showed that above a critical Reynolds number formulated with the pitch line velocity and the face width of the gear, the torque coefficient becomes Re independent, while Fr independence remains. While We number dependence was said to be negligible in the previous two works, it was reported in [17] that aeration of the lubricant can occur in the form of very small bubbles, and gives a simple dimensional engineering model to calculate the resulting increase in power losses, indicating We number dependence. In [18] the authors introduced a special dimensional parameter in order to give a set of better correlations for the different parametric regimes, also possibly indicating $W e$ dependence.

While the hydrodynamic power loss is an important global characteristic of the flow, other, more detailed data may be also needed to validate a flow field obtained by CFD simulation, especially regarding the features where two-phase effects dominate, such as in the case of fluid splashes generated by the gear. Such measurements can be visualizations of the two-phase global flow field or any quantitative field data, such as boundary layer thickness distribution or volume flux of the splashing fluid, injected by the gear.

Unfortunately the literature on these is scarce, however some authors successfully made measurements on simple geometries which can serve as a simplified model of a gearbox flow yet the experimental accessibility is better, providing information on the above mentioned features. The authors of [19] used flash flow field visualizations and PIV on a single smooth gear and a mesh gear partially immersed in fluid in a closed large rectangular tank. Apart from the few shown visualizations, the comparison of momentum thickness distributions in the boundary layer around the immersed parts showed that for large Reynolds numbers, the momentum thickness distribution of a gear and a cylinder do not differ significantly. On a similar geometry, the authors of [20] measured the integrated volumetric flow rates of the fluid sheet splashed by the emerging side of the gear, above the shaft height. The authors used a single gear in a rectangular cavity and collected the splashed fluid by a single container placed in the tank with an entrance window always positioned above the axis height, collecting oil which was ejected by the gear. The authors investigated the influence of geometrical parameters, rotation speed and fluid properties and gave a set of correlations for the flow rates in a semidimensionless form. The authors also explained the key mechanism of the generation of the fluid sheet. According to their theory, the fluid entrained in the boundary layer developing on the submerged part of the gear surface is expelled by the centrifugal forces above the surface, and this material forms the fluid sheet.

Although this basic mechanism seems to be evident from the visualizations of previous authors, comparison of the mass flux delivered by a hypothetical flat plate laminar boundary layer around the gears and the actual mass flux measurements showed significant differences even in the basic trends; however the negative result can come from the laminar boundary layer assumption. On a different geometry, where the laminar assumption is valid, the hypothesis can be applied with success. The subsurface boundary-layer, the surface film flow and the ejected fluid sheet was both successfully characterized experimentally and described quantitatively by [21] around a partially submerged, spinning sphere, and the mass flux measurements were matching with the results of boundary layer theory. The laminar assumption for the boundary layer is also contradicted by the Reynolds-independent torque coefficient measurements by [16] and the later cited results.

\section{Experimental apparatus and methods \\ 2.1 Test rig}

The experimental setup used in our investigations is shown in Fig. 1. The rotating spur gear (1) is situated in the upper tank (2) which is partially filled with the working fluid. The gear is fixed on one end of a mechanical transmission (3) consisting of a shaft supported by two Y-bearing units and a coupling with a DC servo motor (4) providing the driving torque. The rotation rate is precisely controlled by a digital control unit using a 4000-step optocoupler. The elements (5)-(9) (lower reservoir, heater, centrifugal pump, piping, and aquifer) form a hydraulic circuit providing the option of elevating the temperature of the working fluid. During our experiments, the upper tank was disconnected from these parts of the system. The electronics are situated in a dedicated box (10) while the driving signal for the servo motor is generated by a signal generator. The entire setup is built on a rolling rack (11).

We used distilled water as working fluid instead of oil for its perfect optical transparency and for its well known and 
controllable physical properties. While water itself is not a real gear fluid, and has a substantially lower viscosity at room temperature than that of gear fluids, the Reynolds number regime in our experiments $\left(86000<R e_{D}<1000000\right)$ was similar to the experimental conditions of the work of Changenet and coworkers [18] $\left(8800<R e_{D}<1200000\right)$. Moreover, water is thought to be a suitable reference fluid for validating computational models. The setup has been frequently cleaned with alcohol and refilled with fresh distilled water in order to remove any adsorbed film from the liquid-gas interface which could alter surface dynamics, especially surface tension and dissipation of surface waves.

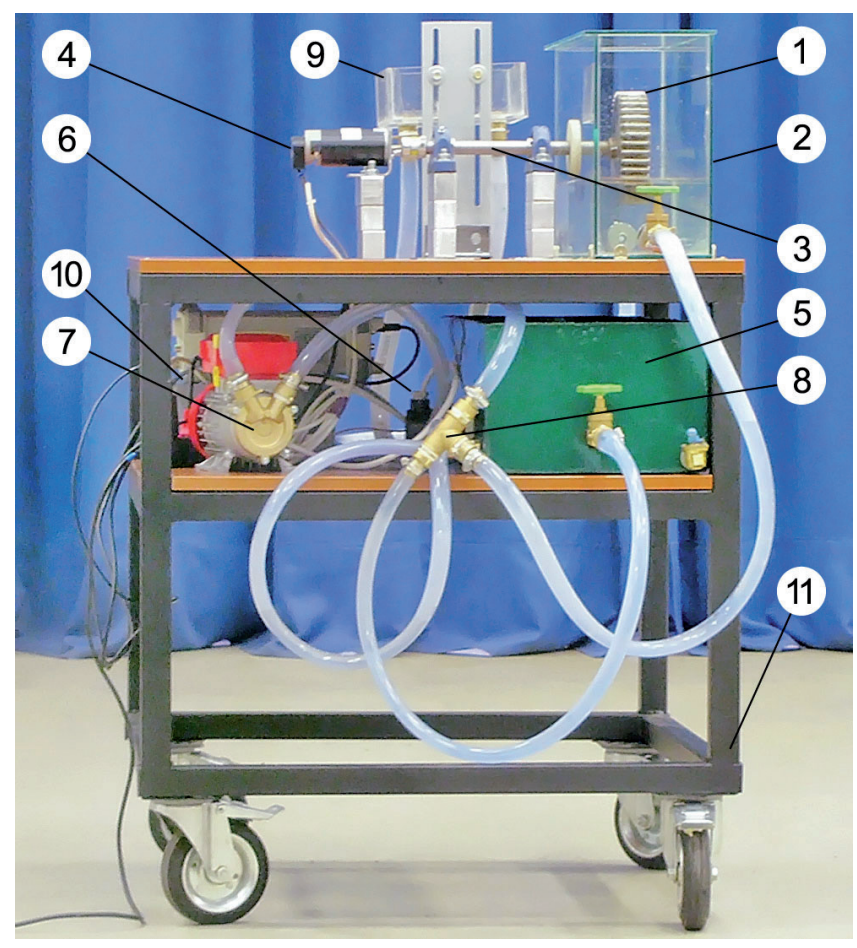

Fig. 1 Picture of experimental setup. For identification of different functional units see main text

\subsection{Mass flow distribution measurement}

The mass flow distribution has been measured using a small container placed in the free jet, intercepting flying water particles on their path and capturing them by a rectangular vertical window. We call this measurement method in-situ bucketing. The measurement procedure was as follows. Before the measurement, the measurement container was made empty and installed in its frame positioning it horizontally and vertically in the tank. The container window was covered with a vertical plate, enabling to turn gear rotation on and to reach steady flow state without collection of water. When the flow became steady, the metal plate was quickly drawn out and the jet started to fill the container. The time needed to fill the container between a minimal and a maximal marked level was measured manually using a chronometer. In order to reduce the human error, the measurements were done by a single person at one session. This method could be later improved by taking high definition vido records and analysing them.
The mass flow rate was calculated as the sum of collected water masses $M_{i}$ over the sum of collection times $t_{i}$ :

$$
q_{M}=\frac{\sum_{N} M_{i}}{\sum_{N} t_{i}} .
$$

We carried out uncertainty quantification of the derived mass flux using classical uncertainty propagation on the first order Taylor-series expansion of the mass flux function with collected mass and measurement time assumed as independent variables. For both, we estimated uncertainties by carrying out five consecutive measurements with some selected settings then calculating mean and empirical variances of the collected mass and measured time. This led to a constant expected value and uncertainty for the mass measurements. The time uncertainty model was found to be fit with a logarithmic function of the expected value. Systematic errors were minimized by requiring the observer to start the time measurement when the water reached a lower level mark already higher than zero, and to stop at a higher level, therefore reaction time and systematic level reading errors were subtracted from each other. Backflow from the top of the tank was reduced by inclining the top of the tank and intrusiveness or upstream influence of the measurement container to the flow was minimized by the sharp and thin leading edges of the window walls.

\subsection{Visualizations}

For the comparative flow visualization photographs, we used a digital camera on a tripod. During the laser sheet visualizations, 1 second exposure time photographs were taken using two $100 \mathrm{~mW}$ laser sheet sources for illumination, aligned with the vertical symmetry plane of the gear. During the flash visualizations, a 4 Joule Xenon flash stroboscope with a single flash per exposure was used.

\subsection{Experimental parameter matrix}

During the experiments reported herein, only the water level and the gear rotation speed was varied. Figure 2 illustrates and Table 1 gives all the relevant geometrical parameters of the gear, tank and the physical parameters of the environment and the working fluid under which the experiments were carried out. For the mass flow measurements, the $h / m=3$ immersion depth was selected and the measurements were done at 2.25, 3, 4,5 and $61 / \mathrm{s}$ rotation frequencies. The measurement plane was located $100 \mathrm{~mm}$ from the left wall, and $X=150 \mathrm{~mm}$ from the center of the gear. The lowest measurement location was close to the water level and the highest close to the upper wall of the tank. For the comparative visualizations, the immersion depth of the gear was chosen to $h / m=3,4$ and 5. At each of the three water level settings, the following frequencies have been used: $1,2,3,4,5,6,7,8$ and $121 / \mathrm{s}$. 


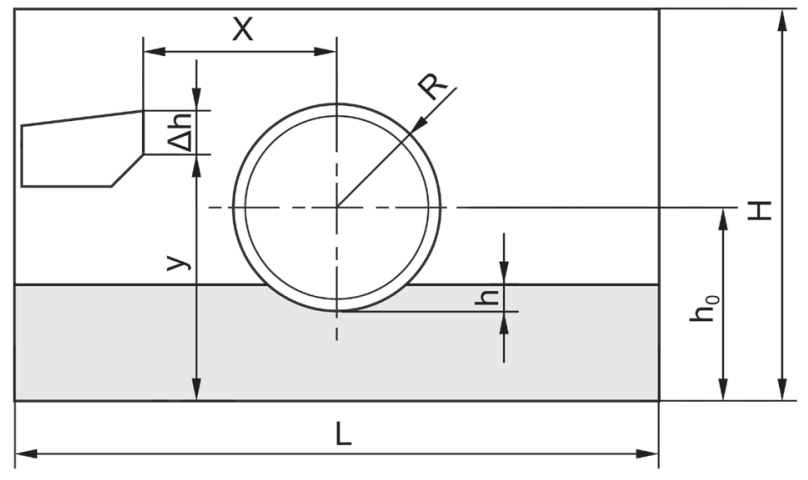

Fig. 2 Schematic sketch of tank, gear and measurement container with relevant geometrical parameters indicated

Table 1 Physical parameters of the setup

\begin{tabular}{|c|c|c|c|}
\hline \multicolumn{4}{|c|}{ Tank: uncoated aquarium borosilicate glass } \\
\hline$H$ & Height & $304.0 \pm 1$ & $\mathrm{~mm}$ \\
\hline$W$ & Width & $150.5 \pm 0.5$ & $\mathrm{~mm}$ \\
\hline$L$ & Length & $500.0 \pm 1$ & $\mathrm{~mm}$ \\
\hline$h_{0}$ & Shaft axis height & $150.0 \pm 0.25$ & $\mathrm{~mm}$ \\
\hline \multicolumn{4}{|c|}{ Spur gear: metric, primer and black acrylic spray coating } \\
\hline$R$ & Tip radius & $80.25 \pm 0.25$ & $\mathrm{~mm}$ \\
\hline$w$ & Width & $40.25 \pm 0.25$ & $\mathrm{~mm}$ \\
\hline$m$ & Module & 4.0 & $\mathrm{~mm}$ \\
\hline \multirow[t]{4}{*}{$Z$} & Number of teeth & 38 & - \\
\hline & Pressure angle & $20^{\circ}$ & \\
\hline & Pitch diameter & 152.0 & $\mathrm{~mm}$ \\
\hline & Root diameter & $142.0 \pm 0.5$ & $\mathrm{~mm}$ \\
\hline \multicolumn{4}{|c|}{ Working fluid: distilled water } \\
\hline$t$ & Temperature & $290.15-293.15$ & $\mathrm{~K}$ \\
\hline$\rho$ & Density [22] & $0.998774-0.998203$ & $\mathrm{~kg} / \mathrm{m}^{3}$ \\
\hline$\eta$ & Dynamic viscosity [23] & $1.0761 \cdot 10^{-3}-8.988 \cdot 10^{-4}$ & $\mathrm{~kg} /(\mathrm{m} \cdot \mathrm{s})$ \\
\hline$\sigma$ & Surface tension [23] & $0.0732-0.0728$ & $\mathrm{~kg} / \mathrm{s}^{2}$ \\
\hline \multicolumn{4}{|c|}{ Environment } \\
\hline$t_{a}$ & Air temperature & $291.15-294.15$ & K \\
\hline$g$ & Gravity & 9.81 & $\mathrm{~m} / \mathrm{s}^{2}$ \\
\hline
\end{tabular}

\subsection{Dimensionless groups}

We describe our experiment by the following selected subset of dimensional parameters: $R, h, X, m, \omega, g, \rho, v$ and $\sigma$. The circumferential velocity of the gear is calculated as

$$
u=R \omega
$$

where $R$ is the tip radius of the gear.

The semivertex angle of the immersed section of wheel $\alpha$ is calculated from the immersion depth $h$ as

$$
\alpha=\arccos \left(1-\frac{h}{R}\right)
$$

and with this, the immersed arc length $s$ is calculated as

$$
s=2 R \alpha .
$$

The following formulation of dimensionless groups is proposed. The Froude number is defined as

$$
F r=\sqrt{\frac{R \omega^{2}}{g}}
$$

capturing the significance of centrifugal forces compared to gravitational force.

The physical significance of the immersed arc length as well as viscosity is captured by the Reynolds number defined with the immersed arc length of the tip cylinder, and the viscosity of working fluid:

$$
R e=\frac{v s}{v}=\frac{R \omega s}{v} .
$$

The effect of surface tension both in the immersed and in the upper part of the flow field is described by the Weber number:

$$
W e=\frac{p_{d y n}}{p_{\text {surf }}}=\frac{\rho R^{2} \omega^{2}}{\frac{\sigma}{m}}=\frac{\rho R^{2} \omega^{2} m}{\sigma} .
$$

The Weber number is viewed here as the ratio of dynamic pressure $\left(p_{d y n}\right)$ and the pressure jump $\left(p_{\text {surf }}\right)$ on the phase interface of a bubble (or droplet) with a characteristic size commensurable to the module size $m$.

Our formulation of dimensionless groups differs from previous works that we propose to use the global length scale $R$ in the $\mathrm{Fr}$ number, for the global phenomena is primarily affected by gravity, while for the Re number we use the immersed arc length $s$ instead of $R$, and instead of neglecting the effect of $W e$, the proposed formulation of the We number uses the peripheral velocity of the gear and the density and surface tension of working fluid and, most importantly, the gear module size as the characteristic length scale of the tooth geometry, which primarily influences interfacial phenomena. Nevertheless exact similarity rules imply identical tooth geometry paremeters, such as adendum and dedendum.

In our experiments, fluid properties and geometry (except immersion depth) were kept the same, therefore two dimensionless numbers - dependent only on geometry and fluid properties but not on speed - were also constant for each immersion depth. One possible choice for these are the Ohnesorge and Eötvös numbers.

$$
O h=\frac{\sqrt{W e}}{R e}=v \sqrt{\frac{\rho}{\sigma} \frac{m}{s}}, \quad E \ddot{o}=\frac{W e}{F r^{2}}=g \frac{\rho}{\sigma} R m .
$$


This also means that during our experiments, there was a proportional relationship between $F r, R e$, and $W e$ :

$$
W e=O h^{2} \cdot \operatorname{Re}^{2}=E \ddot{o} \cdot F r^{2} .
$$

During the discussion of our visualization results, we choose to mention the dependence of the flow pattern on the dimensionless number we felt physically more appropriate to compare the results with, however our choices are arbitrary as the above relations must be taken into account. At the discussion of quantitative results however, we argued which dimensionless number is appropriate as it clearly follows from dimensional analysis and our quantitative results.

\section{Results and discussion}

\subsection{Laser sheet and flash visualization of the typical global flow field}

The features found in a setting typical in our parameter matrix are shown in Fig. 3. The water surface (1) divides the global flow field into an upper part (2) dominated by the gas phase and a lower (3) part dominated by the liquid phase. We find the fluid flow in two different forms around the gear: the thick, unstable and dense ejected liquid sheet (4) and the low-density atomized region characterized by droplets (5). The gear boundary layer (6), visualized by the entrained air bubbles, is the exciting motor of the whole flow. In the vicinity of the emerging point of the wheel, the matter in the boundary layer is dragged above the water surface, where it separates from the gear and continues its path as the liquid sheet. A weak subsurface continuation of the boundary layer ( 7 ) is indicated by some bubbles from the boundary layer traveling forward just below the surface. The fluid sheet splashes on the vertical wall facing the emerging side of the gear and forms the primary wall flow (8). The same splashes occur with lower intensity, forming secondary wall flows at the upper (9) and opposite vertical walls (10). The primary wall flow reenters into the pool, turns back towards the gear and forms a back-flow (11). This back-flow feeds the boundary layer with fresh liquid, therefore the boundary layer, the fluid sheet, the primary wall flow and the back-flow form a closed stream tube. The flow directions in this closed stream tube are visualized by the entrained bubbles in the long exposure photographs. The bubbles entrained at the by the boundary layer continue their way entrapped in the fluid sheet, some of the largest bubbles still present in the re-entrant wall flow and in the back-flow. Likewise the pathlines of the droplets in the atomized region are clearly seen. Sometimes we see falling droplets from the secondary wall flows hitting the gear or the water surface (12). A good indication of the closed stream tube is that at large Weber numbers the backflow region of the lower part becomes opaque due to very small bubbles (13) while the remaining region of the lower part remains clearer. As very small bubbles follow streamlines with very good accuracy, this shows that streamlines do not connect the left and right part of the tank.
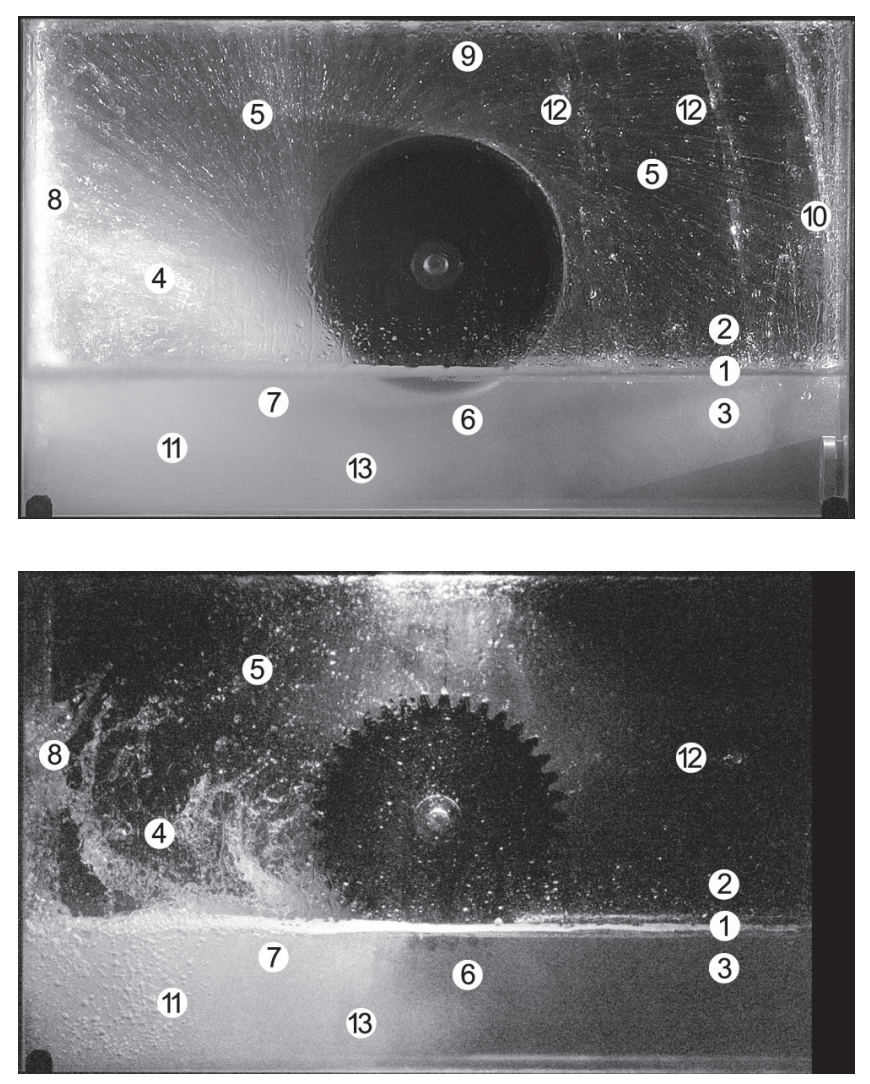

Fig. 3 Identification of typical flow features on laser sheet (above) and flash visualizations (below). Rotation clockwise. $h / m=4, F r=3.41, W e=499, \operatorname{Re}=2.9210^{5}$

\subsection{Flash visualization of the typical boundary layer}

In Figure 4, a typical appearance of the boundary layer of the immersed part of the gear is shown. The rotation of the gear is clockwise, so a tooth submerges in the fluid at the right side. The boundary layer is clearly two phase as many air bubbles are entrained in the liquid phase. It is interesting to see that typically most of the air bubbles reside around the curved side of the gear, while around the flanks only a very few can be seen.

Another important phenomenon which can be clearly seen on almost all photographs is that as the teeth submerge after each other from the air into the water, the gaps between them remain filled with air, and these air gaps are stable.

It is interesting to compare the driving mechanism of the boundary layer on the whole flow in case of our experiments and in case of gear pumps and water turbines. Opposed to the flow in a gear pump, here the tooth gaps are not filled with the liquid, therefore the working principle of volume conservation can be rejected in our case. The excitation mechanism then must be momentum exchange between the gear and the fluid. Together with the solid surface of the gear, the phase boundary of these gaps forms an almost perfect cylindrical boundary which is stable, and impermeable for the fluid. This means that the streamlines are parallel with the wall in the vicinity of the boundary, therefore teeth do not act like individual blades on a water turbine, where momentum exchange is done by pressure gradients during blade impact. We arrive to the conclusion that our system 
behaves like a viscous pump and the momentum exchange between the gear and the fluid is done by the shear stresses.

In our experiments the flanks of the gear and the tips of the teeth are not covered with air and remain wetted by the fluid. This interleaving air-solid surface weakens the no-slip boundary condition on the boundary layer, and imposes a periodic slip-no slip boundary condition instead. One may assume that the weakened boundary condition significantly reduces the driving efficiency of the gear compared to a viscous pump of full solid cylinder. This is not the case, as measurements by other authors, like [19] and [20] show that for large speeds, the boundary layer momentum thickness and the driven mass flow does not differ significantly between a gear with teeth and a smooth, solid cylinder which has the same width and diameter as the gear tip diameter.

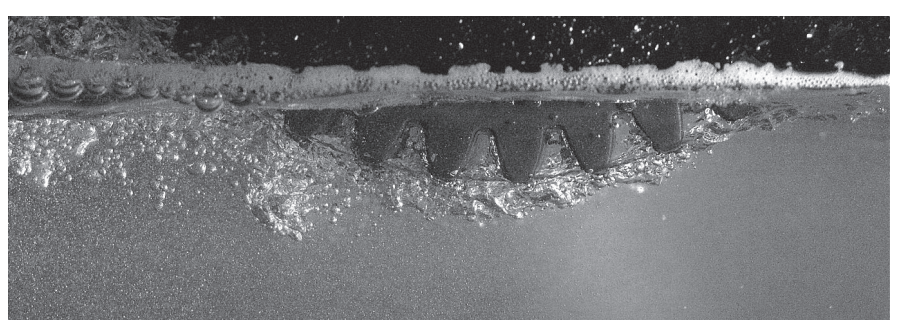

Fig. 4 Close-up of the boundary layer on the immersed part of the gear. Rotation clockwise. $h / m=4, F r=2.27, W e=222, \operatorname{Re}=1.6810^{5}$

\subsection{Variation of boundary layer structure with We}

On Figure 5 we can follow how the air cavities and the two phase layer forms with increase of $W e$ number. At $W e=0$, there are already small bubbles trapped between the submerged teeth. At $W e=13.9$, we find several elliptical bubbles in each tooth gap and almost no bubbles in the boundary layer. At $W e=55.4$, there are incomplete cavities on the leeward side of the teeth with several irregular bubbles in some tooth gaps as well as bubbles around the gear flank. At $W e=125$, the air cavities are completely filling up the tooth gaps, the bubbles travel around the flanks as well as in the side boundary layer, which is intermittently populated with bubbles. From $W e=222$, bubbles around the flank and the intermittent presence of bubbles in the side boundary layer disappear. Despite the 9-fold increase in speed, there is no dramatic difference in the shape and thickness of the boundary layers between $W e=222$ and $W e=2000$. The observable change in the established mixing layer due to We number is that the bubbles become smaller and more evenly distributed with increasing $W e$. The left side of the images for $W e>679$ becomes grey due to very small bubbles convected by the backflow which make the fluid opaque, making the gear and the bubbles in the mixing layer seemingly disappear. There was no noticeable difference between the boundary layers for the same $W e$ for different immersion depths in our experiments.

\subsection{Global flash visualizations and evolution of fluid sheet with We number}

While its time-averaged shape and extent can be seen on the laser photographs, the morphology of the fluid sheet can be seen on the flash visualizations in Fig. 6 and 7. Below $W e=55.4$, a thick and stable fluid sheet takes place at the emerging side of the gear. At $W e=125$, the sheet is already unstable and ligaments are formed on the upper edge of the sheet, atomizing it to droplets. Because of this, an overlap region between the droplets coming from the atomizing fluid sheet and the droplets coming from the teeth appears and remains to be present for higher speeds. From this $W e$ on, the ligaments become larger in vertical extent, thicker, and the whole sheet becomes more opaque due to large entrapped bubbles. From $W e=887$, in the overlap region close to the gear, a periodic pattern appears, possibly indicating separation of fluid from the gear in the form of ligament-type instability.

\subsection{Variation of atomized region with Fr-We and immersion depth on laser sheet visualizations}

The visualizations can be seen in Fig. 8 and 9. The atomized region appears between $W e=55.4$ and 125 , when fluid starts to be ejected from the teeth due to the breakup of bubbles trapped between the teeth, visible in low speed photographs. From that point, pathlines of the individual droplets are clearly visualized except for the region where the fluid sheet takes place. At $F r=1.70$ the atomized region occupies both sides and the space above the gear for all investigated immersion depths. The shape and extent of the atomized region correlates well with the speed and immersion depth of the gear, the smaller immersion depth resulting generally in wider as well as taller extent, while increasing speed resulting in larger extent in both directions. It is interesting to see that the pathlines can be traced back to the gear perimeter where they are started being tangential to the gear surface. It is also noticeable that as the gear speed increases and droplets have increasing speed and kinetic energy, the pathlines converge to straight lines. This can be explained with a simple oblique free fall in the gravity field as well as with the fact that for large Fr numbers, the influence of gravity on pathlines vanishes. For the largest Fr numbers, the pathlines form a plethora of different intensities. The slope of the edge of the largest intensity region, that is, the fluid sheet is around the slope of the tangent to the emerging point of the gear, increasing with increasing immersion depth. Again, the visualization of boundary layer by entrained bubbles and the opacity of the backflow region for large $F r$ are clear. 


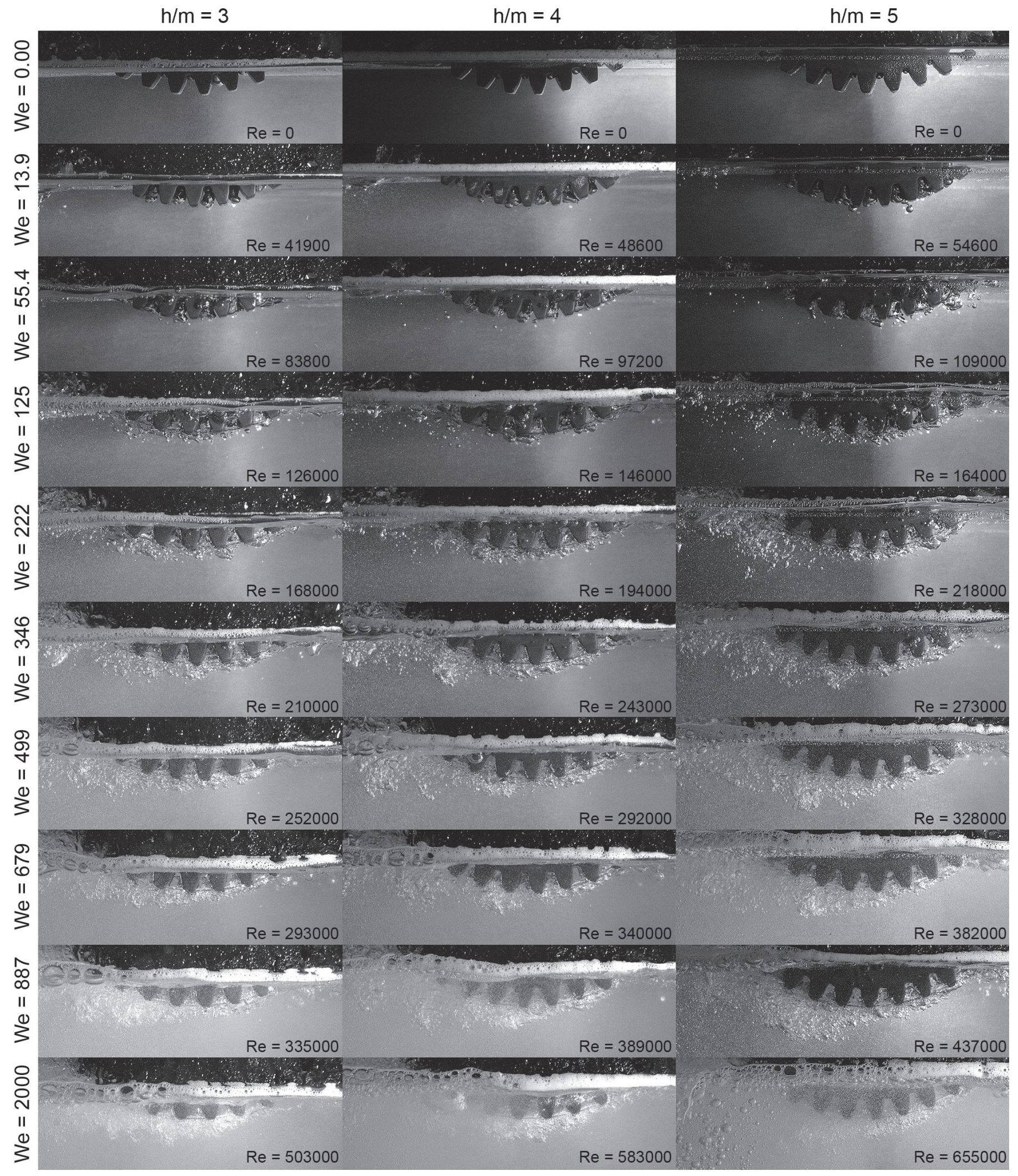

Fig. 5 Instantaneous view of boundary layer of gear for immersion depth of 3, 4 and 5 modules (left, middle and right columns) and for different cycle frequencies (rows): $0,1,2,3,4,5,6,7,8$ and 12 1/s. Rotation clockwise. 


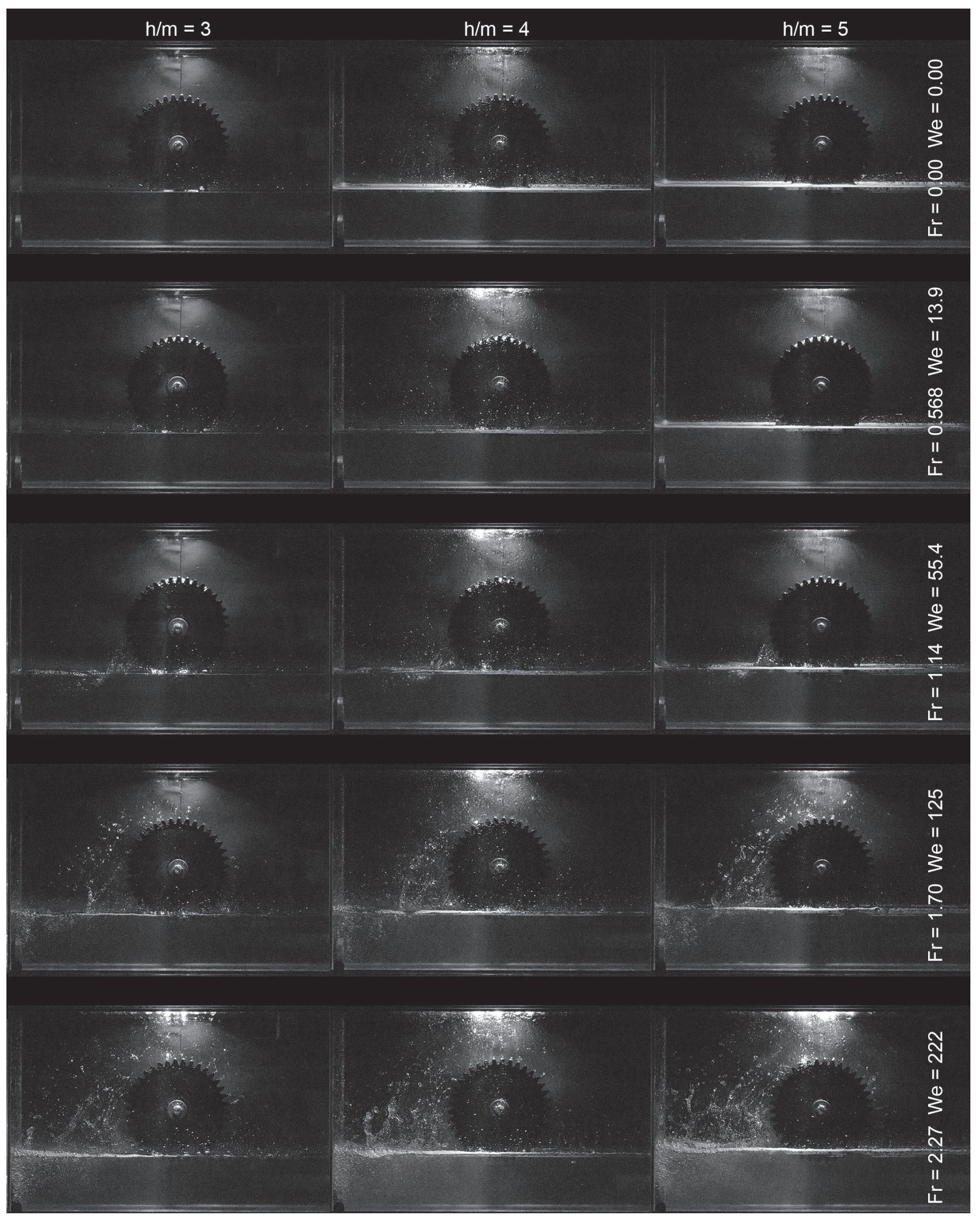

Fig. 6 Instantaneous view of whole flow field for immersion depth of 3, 4 and 5 modules (left, middle and right columns) and for different cycle frequencies (rows): $0,1,2,3$ and 4 1/s. Rotation clockwise. 


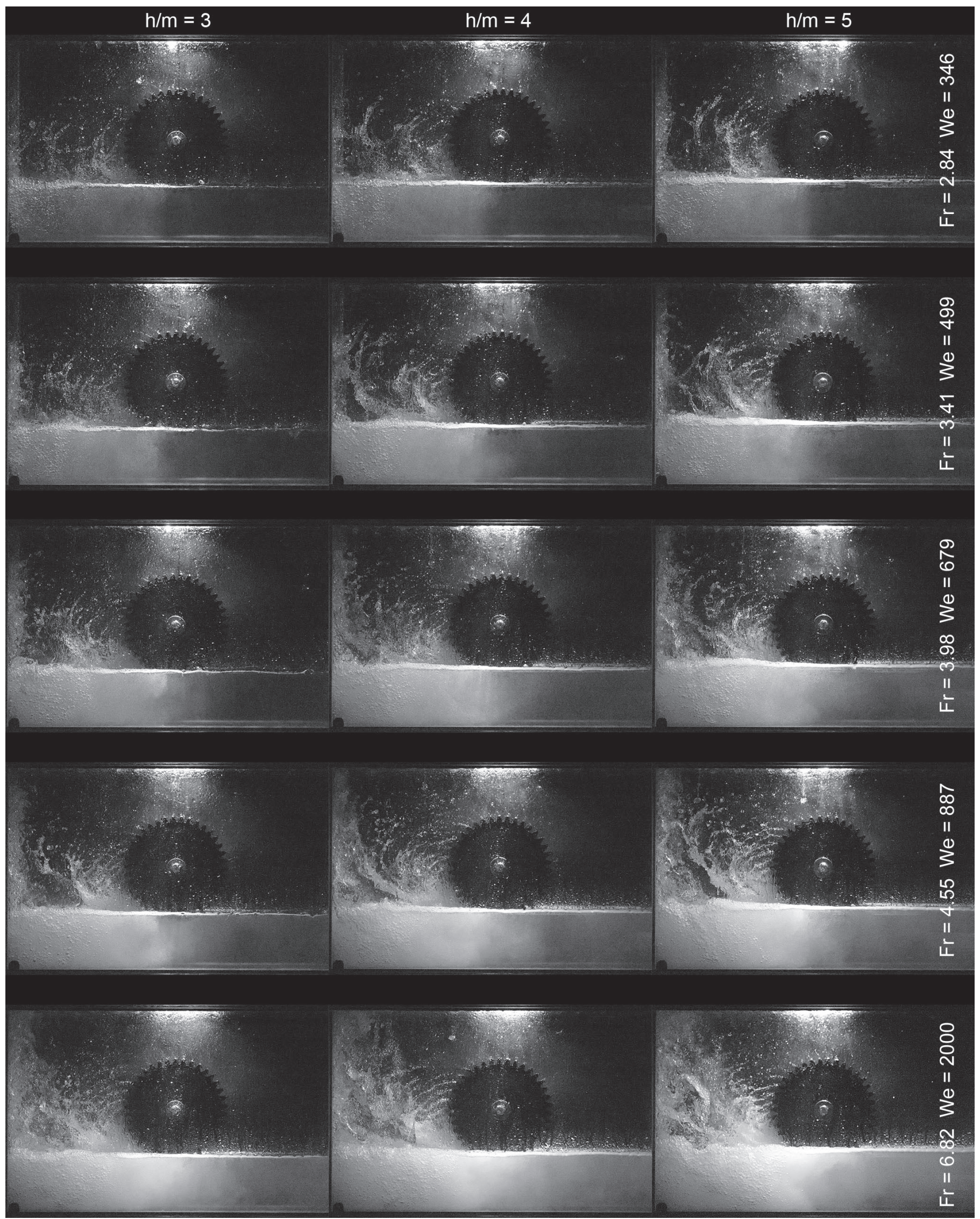

Fig. 7 Instantaneous view of whole flow field for immersion depth of 3, 4 and 5 modules (left, middle and right columns) and for different cycle frequencies (rows): 5, 6, 7, 8 and 12 1/s. Rotation clockwise. 


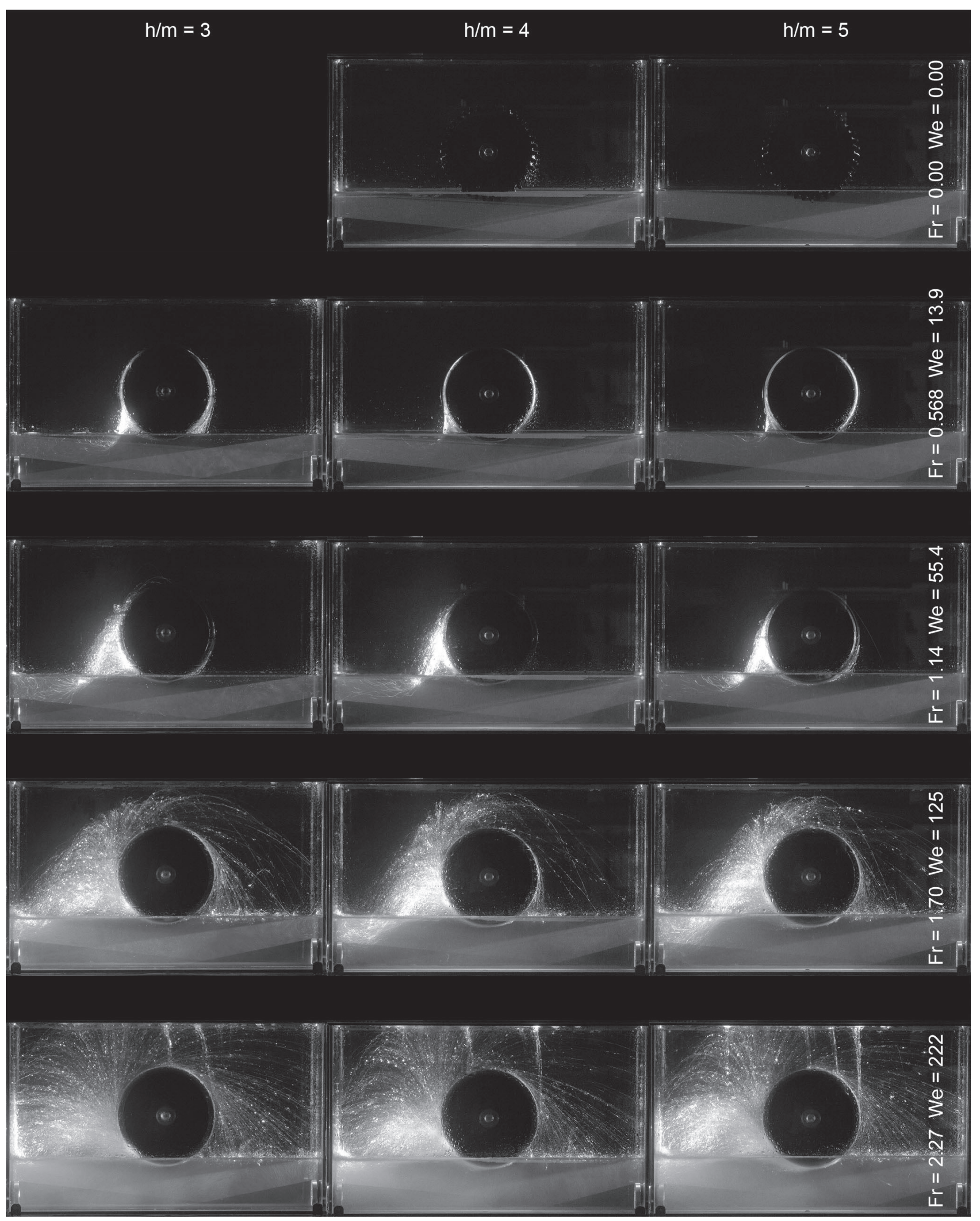

Fig. 8 Laser sheet visualizations of the whole flow field for immersion depth of 3, 4 and 5 modules (left, middle and right columns) and for different cycle frequencies (rows): $0,1,2,3$ and 4 1/s. Rotation clockwise. 


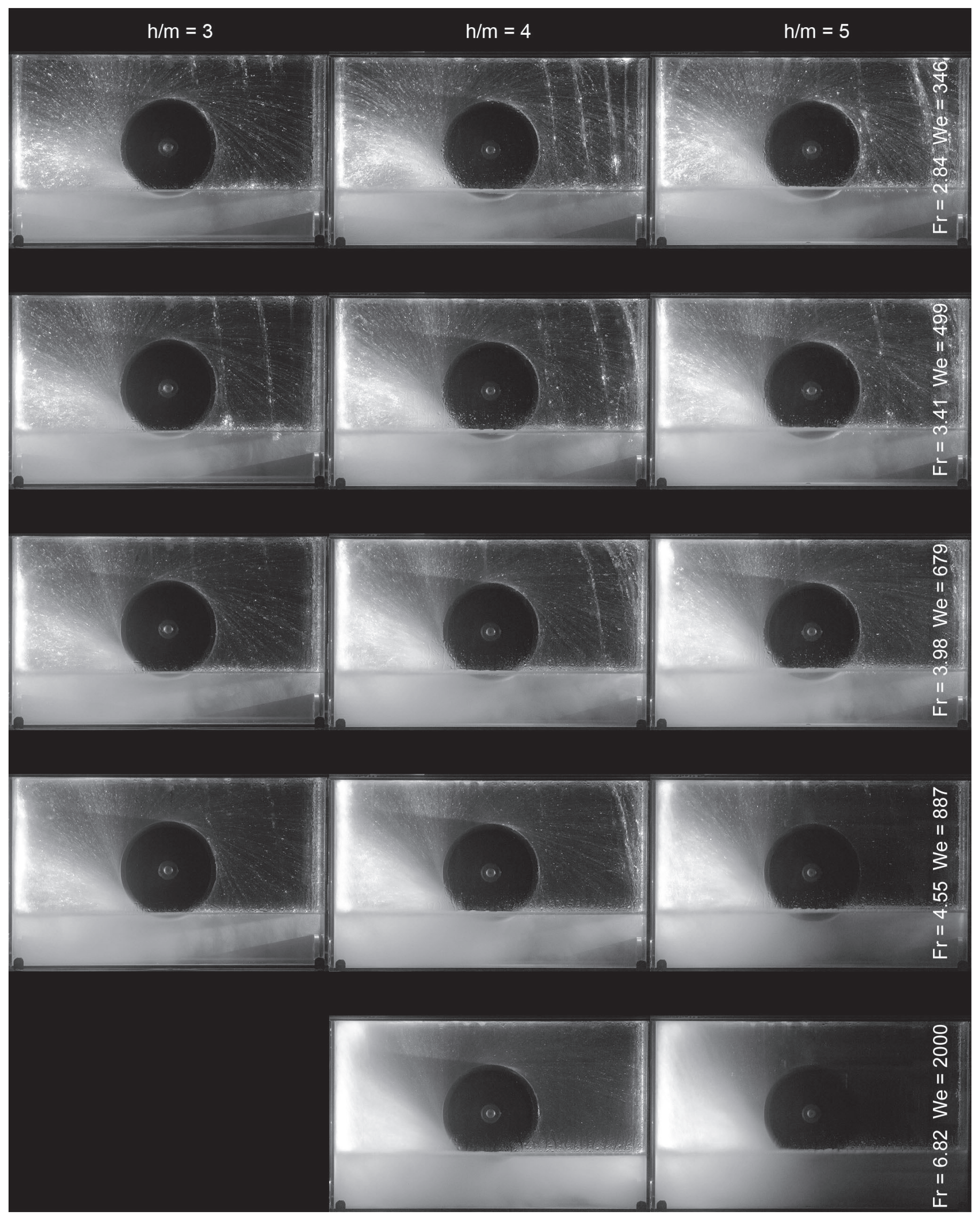

Fig. 9 Laser sheet visualizations of the whole flow field for immersion depth of 3, 4 and 5 modules (left, middle and right columns) and for different cycle frequencies (rows): 5, 6, 7, 8 and 12 1/s. Rotation clockwise. 


\subsection{Mass flow distributions for different velocities and comparative flow visualizations}

Figure 10 shows measured mass flow distributions delivered by the fluid sheet and the atomized region compared using long exposure laser sheet and instantaneous flash photographs (inverted) for increasing $\mathrm{Fr}$ numbers. The vertical line on the photographs marks the measurement plane location.

For the two lowest Froude numbers, the quantitative measurements and the photographs agree in the local vertical extent of the free jet, above which zero mass flux has been measured. The measured distributions have their maximum close to the water level, in the region which has been identified by its morphology as the fluid sheet, while above the maxima, a continuous decay is found in the atomized region. As we can see on the measured distribution, the transition between the atomized region and the fluid sheet is gradual, indicating an overlapping region between them both quantitatively and in behavior. As the pathlines of the two highest $\mathrm{Fr}$ number pictures are close to straight lines, we may expect an asymptotic behavior of the tails of the distributions.

\subsection{Integrated mass flux measurements and Re-independence}

Integrating the measured mass flux distribution along the measurement plane from water level to the tank top for and plotting the resulting mass fluxes against rotation speed we get the diagram on Fig. 11. The linear function is found to fit with remarkably good accuracy. The only outlier (omitted from fit) is the first measurement point which was found where a few of the most far-reaching droplets just started to reach the container, thus marking the speed where the fluid sheet reached the measurement pane. This happened at the lowermost point of the measurement plane, that is, at the water level. We will call this speed the critical speed. During this series of measurements, all other parameters were fixed except for the gear speed. Therefore this experiment shows the influence of gear speed only on integrated mass flux, separated from the influence of all other parameters. Let us consider what implications the measured functional form of mass flux-gear speed function bear when dimensional analysis is considered. Our function can be described quantitatively by three values: the critical speed $\omega_{c}$; the intercept speed of the asymptote line $\omega_{0}$ which can be considered an approximation of the critical speed itself; and the slope of the line, $A$.

The limiting frequency and the intercept are two fixed speed values. Therefore, while these parameters can be functions of the parameters fixed in our experiment, it is clear that none of these functions can have the gear speed, $\omega$ itself, as an independent variable:

$$
\omega_{c}=f_{1}(R, h, X, \ldots, \rho, v, \sigma, g), \frac{\partial \omega_{c}}{\partial \omega}=0,
$$

$$
\omega_{0}=f_{2}(R, h, X, \ldots, \rho, v, \sigma, g), \frac{\partial \omega_{0}}{\partial \omega}=0 .
$$

This also means that if we apply dimensional analysis on these functions to find their dimensionless forms, when finding a suitable dimensional scale of speed, we must rule out rotational velocity. This leaves us with gravity $g$, kinematic viscosity $v$ and surface tension $\sigma$ containing time scale. Picking any of the allowed choices, after nondimensionalization, we arrive to a function in which none of the dimensionless parameters contain velocity. Since simple algebraic rearrangement of these dimensionless functional equations must give back their dimensional form, in which $\omega$ did not appear as an independent variable, it means that introducing some power of $\omega$ at both sides is mathematically allowed however such an alternative form can be always rearranged to a dimensionless form in which only dimensionless numbers independent of $\omega$ appear.

This means not only that the dimensionless critical speed and the dimensionless asymptotic speed intercept are independent of Reynolds number, but also from Froude and Weber numbers. The independent dimensionless variables of these functions depend, therefore, only on the setup geometry (including measurement plane) and the properties of the fluid. Apart from the geometric ratios, we can make two of such dimensionless numbers from our original set of dimensional parameters. One possible set is the Ohnesorge and Eötvös numbers.

After stating these general but strong considerations, let us try to formulate a concrete functional from of these speeds first in dimensional then in dimensionless form. We noticed that the mass flux at the critical speed consisted of small individual droplets flying on ballistic arcs. We assume that the starting point of these particles is the emerging point of the gear while the speed is equal to the circumferential speed, and the initial path angle is the tangent angle of the emerging point. Since the speed of the droplets was low, we neglected drag and kept gravity only, and determined the pathline of the droplets as the parabolic arc under the influence of gravity only. With these assumptions, the horizontal distance traveled by a droplet becomes:

$$
x=\frac{R^{2} \omega^{2}}{g} \sin 2 \alpha .
$$

And from this, we find the critical speed when $\mathrm{x}$ is equal to the distance between the emergence point and the measurement plane calculated as $x_{m}=X-R \sin \alpha$ :

$$
\omega_{c}=\frac{g x_{m}}{R^{2} \sin 2 \alpha} .
$$



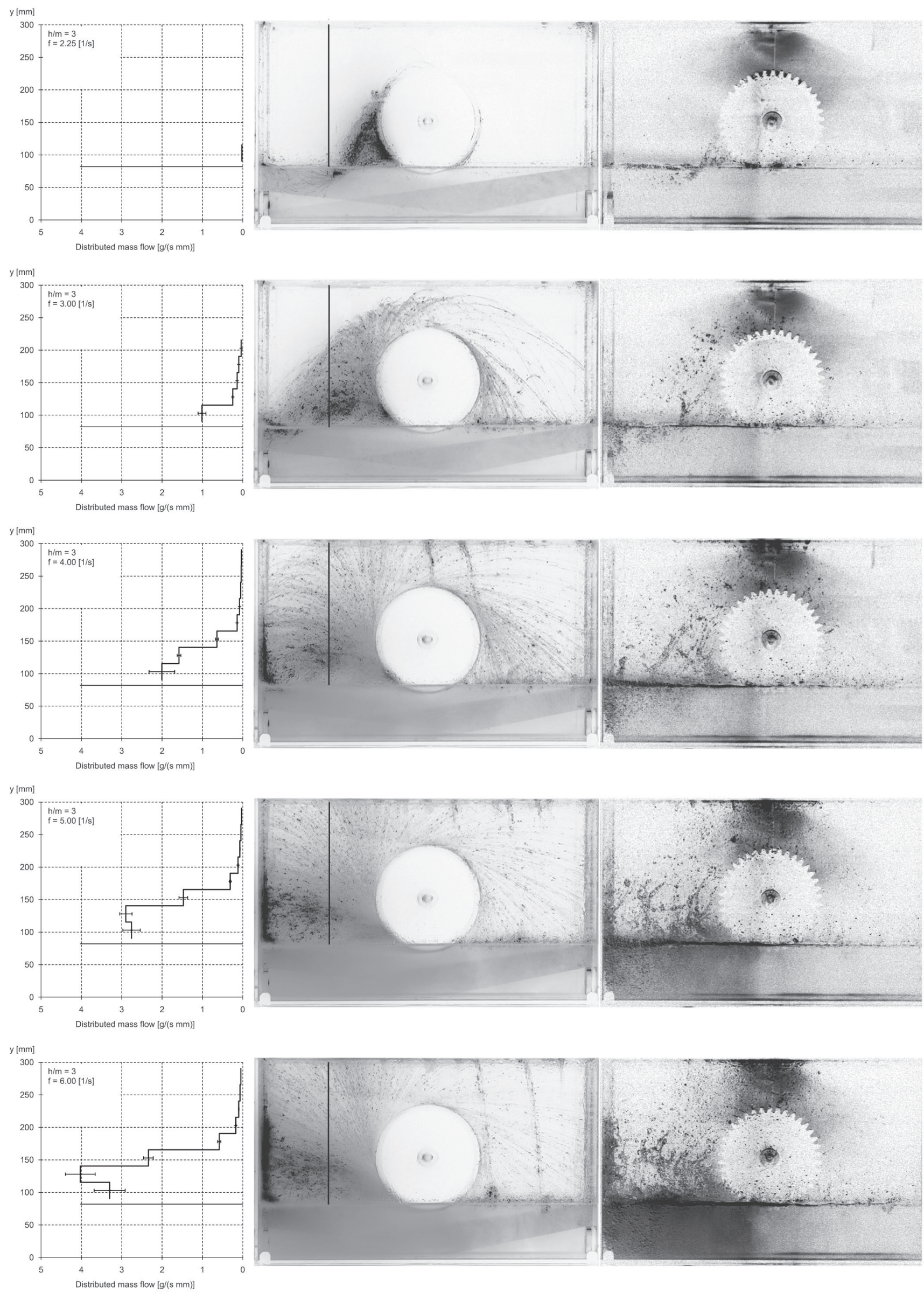

Fig. 10 Measured mass flow distributions delivered by the free jet compared with long exposure laser sheet and instantaneous flash photographs for different cycle frequencies. Vertical line on photographs marks measurement plane location. We $=70.2,125,222,346,499$ from top to bottom, respectively. 


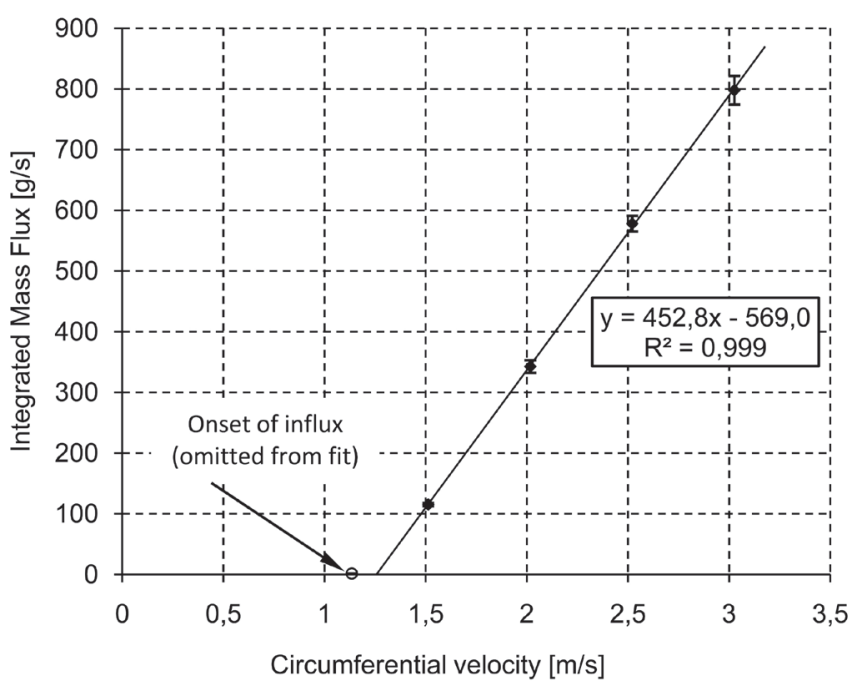

Fig. 11 Measured mass flow distributions delivered by the free jet integrated on the whole measurement section as a function of circumferential speed of the gear. A linear function (omitting the first point) fits data remarkably.

The numerical calculation of the critical speed based on the model gives $13.546 \mathrm{rad} / \mathrm{s}$ while the actual measurement is $14.137 \mathrm{rad} / \mathrm{s}$ which can be considered a good agreement. We note that the bucket edge was slightly above the surface in this experiment for avoiding false flux from wave splashes, nevertheless the elevation was no more than $5 \%$ of the distance from the ejection point and comparable to the droplet size. Therefore we neglected the bucket elevation in Eq. (13).

When finding the nondimensional form of the above equation, we see that our only possible choice to form a dimensionless number is to use the gravity as the natural speed scale, resulting in a critical Froude number criterion:

$$
F r_{c}=\sqrt{\frac{x_{m}}{R} \sin 2 \alpha} .
$$

We see that according to the physical model, the critical Froude number depend only on kinematic conditions, and not on fluid properties, therfore $F r_{c}$ does not depend either on $R e$ or $W e$. In fact it is even independent from the fluid properties that is, from $O h$ and $E \ddot{o}$.

In case of finding a physical model to the intercept speed correlation, the argument can be similar to the one for the critical speed however in this case the parabolic pathline should belong to the limiting path of the thick and contiguous jet. A correction for the real fluid properties of the jet is necessary:

$$
F r_{0}=F r_{c}\left(1+f\left(\alpha, x_{m} / R, \ldots, O h, E \ddot{o}\right)\right) .
$$

Alternatively, a different physical consideration can connect the intercept speed to another, "stronger" critical speed, determined by a potential energy barrier for the flow starting as a subsurface jet to come above the surface. Since such a potential energy barrier would be proportional to $g$ and to some height difference scale such as $h$, following this argument we arrive to a time scale formed with $g$ and a functional form giving a Froude number criterion, leaving the original speed independence argument unchanged.

Now let us turn our attention to the linearity of the measured mass flux as function of gear speed, notably to the constant nature of the slope of the line fit. The linear function may be formulated with the intercept speed as a natural bias as:

$$
q_{M}=A \Delta \omega \text { where } \quad \Delta \omega \equiv \omega-\omega_{0} .
$$

Here the dependence of $q_{M}$ on $\omega$ is explicitly written in the linear term, therefore $A$ must not depend on $\omega$. The question arises again what to choose as time scale. Once we argued for the fact that $\omega_{0}$ is strongly connected to gravity and the choice of the measurement plane distance, subtracting it from the speed, we can argue that $\Delta \omega$ can be considered an independent variable, giving the time scale itself. Now dividing both sides with $\Delta \omega$ gives us a formulation where only $A$ stands on the right side as a function of independent dimensional variables, $\omega$ excluded again.

$$
\frac{q_{M}}{\Delta \omega}=A(R, h, X, \ldots, \rho, v, \sigma, g), \frac{\partial A}{\partial \omega}=0 .
$$

For finding a proper dimensionless form of the above equation, which also shows the influence of the underlying physical phenomena, we must consider what physical parameters we can use for normalization of $A$.

In order to choose the proper scaling parameter group, we will stand on the experimental findings of [20] as well as our own results.

Firstly, as $A$ has the dimension of $[\mathrm{kg}]$, we must include either $\rho$ or $\sigma$. Since we multiplied our measurement results with $\rho$ only during the postprocessing of the measurements, we must choose $\rho$ here as well, providing $[\mathrm{kg}]$ with the proper first power. With this, $\sigma$ is ruled out, which is also reasoned by the fact that the influence of $\sigma$ is found negligible in [20]. From this, it comes that we must furthermore include one or more of the dimensional parameters containing [m], giving an $\left[\mathrm{m}^{3}\right]$ group together. The selection of these is a key point in our argument, as there are several geometric parameters with the dimension [m] as well as several remaining physical parameters of the fluid and the environment containing [m], and not ruled out by the previous arguments. These are the following: $R, h, X, m$ as well as other geometric parameters describing the gear geometry, such as its with $W$ (not used in the previous discussions). Among the environmental parameters, we have the environmental gravitational force field strength $g$, and the fluid viscosity $v$.

Now, it is evident from our own visualizations as well as from the previous work that the mass ejection is induced by the gear boundary layer, therefore the measured mass flow rates above the water level must be strongly influenced by the physical dimensions of the stream tube constituting the gear 
boundary layer and the velocity distribution of in this stream tube. The boundary layer is forced and turbulent, and therefore dominated by inertial effects. The descriptive velocity scale is the gear peripheral velocity $R \omega$. To represent this, we must include the tip radius, $R$ in the scaling factor of $A$. It was found in [20] that the mass flux is also directly proportional with very good accuracy to the gear width, $W$, therefore it is reasonable to include it in the scaling factor as well. With these choices, we can rewrite Eq. (16) as

$$
q_{M}=\rho R W h_{1}\left(\omega-\omega_{0}\right)
$$

which is recognized as a simple but descriptive model for the mass flux, with $R\left(\omega-\omega_{0}\right)$ the effective average velocity and the product $W h_{1}$ being the effective area of the rectangular stream tube. The introduced new quantity $h_{1}$ with dimension $[\mathrm{m}]$ is defined as the effective displacement thickness "responsible" for the mass flux above the water level, at the measurement distance from the emerging point of the gear. Combining Eq. (17) and (18), the linearity of our measurements can be now stated as

$$
\frac{\partial h_{1}}{\partial \omega}=0
$$

Indeed, we have shown before that our choice for the scaling factors are motivated by experimental evidence and that these scaling factors, namely $\rho R W$ were constant, then $h_{1}$ must have been also constant during our measurements, during which only $\omega$ changed. Therefore Eq. (18) is the only statement we can have about $h_{1}$. If this is true, then we can also multiply Eq. (18) with any group of parameters which were constant during the measurement, which includes the kinematic viscosity $v$ and any geometrical parameter arbitrary. With this, with an arbitrary normalizing scale for the displacement thickness, our final result is

$$
\frac{\partial \frac{h_{1}}{R}}{\partial \frac{\omega R s}{v}} \equiv \frac{\partial \bar{h}_{1}}{\partial R e}=0 .
$$

That is, during our experiments, the dimensionless effective boundary layer displacement thickness was Reynolds-number independent.

As a final note, the above argument can be used to show that the displacement thickness is also independent of Froude and Weber numbers,

$$
\frac{\partial \bar{h}_{1}}{\partial R e}=\frac{\partial \bar{h}_{1}}{\partial F r}=\frac{\partial \bar{h}_{1}}{\partial W e} 0 .
$$

and, similarly to the dimensionless critical speed, the functional form of the dimensionless displacement thickness can also include only geometric ratios and the dimensionless numbers not including $\omega$, that is, the Eötvös and Ohnesorge numbers:

$$
\bar{h}_{1}=f\left(\alpha, x_{m} / R, \ldots, O h, E \ddot{o}\right) .
$$

\section{Conclusions}

In this work, we have presented measurements in a two-phase flow generated by a single rotating gear in a large rectangular tank, serving as a minimal model for the dip-lubrication process in gearboxes. By using water as a working fluid instead of high viscosity oil, the necessary gear speed in has been reduced in order to investigate the phenomena in the relevant Reynolds number region. High quality flash light and laser sheet visualizations has been presented of the global flow field as well as of the vicinity of the gear for different gear speeds, showing the evolution of the different flow features, which are: the dense ejected liquid sheet above the water surface, and the two-phase boundary layer induced by the gear. It has been shown that the ejected sheet is the continuation of the induced boundary layer and that the momentum exchange between the gear and the fluid is a viscous pump mechanism, driven by the shear stress.

Resolved measurements of the mass flux distribution of the ejected fluid sheet have been done in a vertical plane at a distance from the gear, between the water surface and the tank top, with a novel experimental apparatus.

It was found that the integrated mass flux at the measurement plane is zero below a critical speed and it is linear in the function of gear speed above the critical speed. It has been shown that the critical speed is Reynolds number independent and a simple analytical formula, based on the parabolic path of first droplets reaching the plane, has been derived to predict its value with a good agreement.

The linear mass flux measurements have been explained using a boundary layer stream tube model. Dimensional analysis has been used to find the proper scaling parameters of the mass flux in the boundary layer. As a final result of this analysis, it has been shown that the linearity is equivalent to the Reynolds independency of the boundary layer displacement thickness in our measurements.

\section{Acknowledgement}

This research is performed in collaboration with the Rába Axle Ltd., Győr, Hungary in the frame of a founded project (Rába Axle Ltd. project nr. GOP-1.1.1-11-2012) supported by the European Union. The work has been supported by the Hungarian Research Fund under contract nr. OTKA K081621 (Transport Processes in Multi-Component Bubbly Flows). This work has been developed in the framework of the project "Talent care and cultivation in the scientific workshops of BME" project. The project is supported by the grant TAMOP4.2.2/B-10/1-2010-0009. The work relates to the scientific program of the project "Development of quality-oriented and harmonized $\mathrm{R}+\mathrm{D}+\mathrm{I}$ strategy and the functional model at BME". 
The New Hungary Development Plan (Project ID: TÁMOP4.2.1/B-09/1/KMR-2010-0002) supports this project.

The authors give special thanks to Rába Axle Ltd. and Dr. Márk Lelkes for technical and professional support, to Dr. János Vad and our colleagues at the Department of Fluid Mechanics and to Balázs Tóth and Dr. Gábor Szabó at the Department of Hydraulic and Water Resources Engineering for the fruitful discussions and general support. Thanks to Mahmoud Asali, Réka Havas, Balázs Rosenthal and Gábor Bócz for their assistance in the development of the setup and conducting the measurements.

\section{References}

[1] Gorla, C., Concli, F., Stahl, K., Höhn, B.-R., Michaelis, K., Schultheiß, H., Stemplinger, J.-P. "Hydraulic losses of a gearbox: CFD analysis and experiments." Tribology International. 66, pp. 337-344. 2013. DOI: 10.1016/j.triboint.2013.06.005

[2] Arisawa, H., Nishimura, M., Imai, H., Goi, T. "Computational Fluid Dynamics Simulations and Experiments for Reduction of Oil Churning Loss and Windage Loss in Aeroengine Transmission Gears." Journal of Engineering for Gas Turbines and Power. 136(9), p. 092604. 2014. DOI: $10.1115 / 1.4026952$

[3] Concli, F., Gorla, C., Della Torre, A., Montenegro, G. "Churning power losses of ordinary gears: A new approach based on the internal fluid dynamics simulations." Lubrication Science. 27 (5), pp. 313-326. 2015. DOI: $10.1002 / 1 \mathrm{~s} .1280$

[4] Concli, F., Gorla, C. "Computational and experimental analysis of the churning power losses in an industrial planetary speed reducer." Advances in Fluid Mechanics. 74, pp. 287-298. 2012. DOI: 10.2495/AFM120261

[5] Concli, F., Conrado, E., Gorla, C. "Analysis of power losses in an industrial planetary speed reducer: Measurements and computational fluid dynamics calculations." Proceedings of the Institution of Mechanical Engineers, Part J: Journal of Engineering Tribology. 228(1), pp. 11-21. 2014. DOI: $10.1177 / 1350650113496980$

[6] Ohshima, S.-I., Matsumura, S., Houjoh, H. "Experimental study of the lubricant flow around the mesh-ending region simulated in water using a helical gear pair." Transactions of the Japan Society of Mechanical Engineers, Part C. 78 (785), pp. 240-251. 2012. DOI: 10.1299/kikaic.78.240

[7] Li, L., Versteeg, H. K., Hargrave, G. K., Potter, T., Halse, C. "A study of fluid flow of gear lubrication in a spur gear box." VDI Berichte. 2029, pp. 133-146. 2008.

[8] Lin, Y., Hu, Z., Xiong, C., Zang, M., Jia, Y., Chen, Y., Luo, D., Zhao, F. "Research of Flow Field Simulation for Lubrication System and Effect Evaluation on a 7-Speed Dual Clutch Transmission." In: Proceedings of the FISITA 2012 World Automotive Congress. Vol. 195, pp. 285-298. 2013. DOI: $10.1007 / 978-3-642-33835-9 \_27$

[9] Concli, F., Gorla, C., Della Torre, A., Montenegro, G. "Windage Power Losses of Ordinary Gears: Different CFD Approaches Aimed to the Reduction of the Computational Effort." Lubricants. 2(4), pp. 162-176. 2014. DOI: $10.3390 /$ lubricants 2040162
[10] Yazdani, M., Soteriou, M. C. "A novel approach for modeling the multiscale thermo-fluids of geared systems." International Journal of Heat and Mass Transfer. 72, pp. 517-530. 2014.

DOI: 10.1016/j.ijheatmasstransfer.2014.01.035

[11] Mauz, W. "Hydraulische Verluste von Stirnradgetrieben bei Umfangsgeschwindigkeiten bis $60 \mathrm{~m} / \mathrm{s}$." (Hydraulic losses of spur gears with peripheral speeds up to $60 \mathrm{~m} / \mathrm{s}$.) Bericht des Institutes für Maschinenkonstruktion und Getriebebau. Nr. 159. Universität Stuttgart. 1987. (in German)

[12] Höhn, B.-R., Michaelis, K., Otto, H.-P. "Influences on No-Load Gear Losses." ECOTRIB. 2011.

[13] Gorla, C., Concli, F., Stahl, K., Stahl, K., Höhn, B., Klaus, M., Schultheiss, H., Stemplinger, J. "CFD Simulations of Splash Losses of a Gearbox." Advances in Tribology. 2012, Article ID 616923. pp. 1-10. 2012. DOI: $10.1155 / 2012 / 616923$

[14] Stavytskyy, V., Nosko, P., Fil, P., Karpov, A., Velychko, N. "Loadindependent power losses of gear systems: a review." TEKA. 10B. pp. 205-213. 2010. URL: http://www.pan-ol.lublin.pl/wydawnictwa/ TMot10b/Stavytskyy.pdf

[15] Changenet, C., Velex, P. "A Model for the Prediction of Churning Losses in Geared Transmissions-Preliminary Results." Journal of Mechanical Design. 129(1), pp. 128-133. 2007. DOI: 10.1115/1.2403727

[16] Changenet, C., Velex, P. "Housing Influence on Churning Losses in Geared Transmissions." Journal of Mechanical Design. 130(6), 062603062603-6. 2008. DOI: 10.1115/1.2900714

[17] LePrince, G., Changenet, C., Ville, F., Velex, P., Dufau, C., Jarnias, F. "Influence of Aerated Lubricants on Gear Churning Losses-An Engineering Model." Tribology Transactions. 54(6), pp. 929-938. 2011. DOI: 10.1080/10402004.2011.597542

[18] Changenet, C., Leprince, G., Ville, F., Velex, P. "A Note on Flow Regimes and Churning Loss Modeling." Journal of Mechanical Design. 133(12), 121009-121009-5. 2011. DOI: 10.1115/1.4005330

[19] Chernoray, V., Jahanmiri, M. "Experimental study of multiphase flow in a model gearbox." In: WIT Transactions on Engineering Sciences. Vol. 70, pp. 153-164. 2011. DOI: 10.2495/MPF110131

[20] Leprince, G., Changenet, C., Ville, F., Velex, P. "Investigations on Oil Flow Rates Projected on the Casing Walls by Splashed Lubricated Gears." Advances in Tribology. 2012, Article ID 365414, 2012. DOI: $10.1155 / 2012 / 365414$

[21] Langley, K. R. "Investigations of Partially Immersed Spinning Spheres in a Liquid Bath and Butterfly Flight." Bringam Young University. All Theses and Dissertations. Paper 3948. 2013. URL: http://scholarsarchive. byu.edu/etd/3948

[22] Weast, R. C. (ed.) "CRC Handbook of Chemistry and Physics." 53rd ed. 1972.

[23] Rohsenow, W. M., Hartnett, J. P., Cho, Y. I. (eds.) "Handbook of Heat Transfer." 3rd ed. McGraw \& Hill. 1998. 